\title{
Phase-Transition Energies, New Characterization of Solid Materials and Anisotropy
}

\author{
Gerd Kaupp \\ University of Oldenburg, Oldenburg, Germany \\ Email: gerd.kaupp@uni-Oldenburg.de
}

How to cite this paper: Kaupp, G. (2019) Phase-Transition Energies, New Characterization of Solid Materials and Anisotropy. Advances in Materials Physics and Chemistry, 9, 57-70.

https://doi.org/10.4236/ampc.2019.94006

Received: February 8, 2019

Accepted: April 21, 2019

Published: April 24, 2019

Copyright (อ 2019 by author(s) and Scientific Research Publishing Inc. This work is licensed under the Creative Commons Attribution International License (CC BY 4.0).

http://creativecommons.org/licenses/by/4.0/

\section{(c) (i) Open Access}

\begin{abstract}
Non-iterative analysis of indentation results allows for the detection of phase transitions under load and their transition energy. The closed algebraic equations have been deduced on the basis of the physically founded normal force $\propto$ depth $^{3 / 2}$ relation. The precise transition onset position is obtained by linear regression of the $F_{\mathrm{N}}=k h^{3 / 2}$ plot, where $k$ is the penetration resistance, which also provides the axis cuts of both polymorphs of first order phase transitions. The phase changes can be endothermic or exothermic. They are normalized per $\mu \mathrm{N}$ or $\mathrm{mN}$ normal load. The analyses of indentation loading curves with self-similar diamond indenters are used as validity check of the loading curves, also from calibration standards that exhibit previously undetected phase-transitions and are thus incorrect. The phase-transition energies for fused quartz are determined from the loading curves from instrument provider handbooks. The anisotropic behavior of phase transition energies is studied for the first time. Quartz is a useful test object. The reasons for the packing-dependent differences are discussed on the basis of the local crystal structure under and around the inserting tip.
\end{abstract}

\section{Keywords}

Instrumented Indentation, Loading Curve, Phase Transition Onset and Energy, Iteration-Less Plot, Physical Exponent, Present ISO Standard, Energy Law Violations, Physical Hardness, Error Detections

\section{Introduction}

Instrumented indentations require proper calibrations and physically correct analysis. Modern instruments are highly reliable (at least since 1998) and the most used standards fused quartz, aluminum and sapphire are available in con- 
stant good quality. There are however problems with non-consideration of largely unknown phase transitions at moderately high loads of conical or pyramidal indentations [1] [2]. Even worse is the data treatment according to the ISO14577 standard (of the International Organization for Standardization), accepting the highly acclaimed Oliver-Pharr method [3]. As generally known, this is based on very complicated mathematical deductions that clearly forgot to take into account the sidewise forces and thus energies at conical penetrations. Their deduced normal force $\left(F_{\mathrm{N}}\right)$-depth square $\left(h^{2}\right)$ proportionality [4] [5] is therefore invalid. This standard cannot describe the experimental loading curves. Rather polynomial iterations are used, but these wipe out all surface effects, local gradients, elbows, and phase transitions under load. It was empirically found that the exponent $3 / 2$ on $h$ correctly describes the load parabola. This was first published in 2005 [6], with a review in 2006 [7], and later in [1] and [2]. Equation (1) provided excellent correlations and numerous unprecedented applications without fittings, or iterations. The validity of (1) was physically deduced in a clear-cut way. This was published electronically in 2016 and open access in 2017 [8]. The basic idea for the physical deduction of the exponent $3 / 2$ on $h(1)$ [8] is the undeniable fact that the entire pressure plus pressure work-and thus also the corresponding part of the normal force $F_{\mathrm{N}}$-goes with the indented volume of cones or pyramids, which is proportional to $h^{3}$. This deduction of the physically enforced Equation (1) can easily be repeated with simple arithmetic. Or graphically: the work that is lost for the penetration is the area between the parabola with exponent $3 / 2$ and its secant that starts at zero. Furthermore, the applied work ( $W_{\text {applied }}$ ) is the area under such secant of the parabola (down to the zero line). Furthermore, the indentation work $\left(W_{\text {indent }}\right)$ covers the area below the parabola, and it can also be described by the area of the $0-h_{\max }-0.8 F_{\mathrm{N}}$ triangle [9]. It follows the $5 / 4$ ratio of $W_{\text {applied }} / W_{\text {indent }}$ that was already mathematically deduced by integration of (1) in [10]. The $k$-values in Equation (1) are the validated penetration resistances. They are obtained by linear regression of the $F_{\mathrm{N}}$ versus $h^{3 / 2}$ plots with excellent correlation. This viable analytical tool is disdainfully known as "Kaupp fitting" in the literature. We must therefore call it now "Kaupp plot (1)" to underline that it must not be degraded to a fitting technique.

$$
F_{\mathrm{N}}=k h^{3 / 2}
$$

The $W_{\text {applied }} / W_{\text {indent }}=5 / 4$ relation means that the loss of $F_{\mathrm{N}}$ for the penetration depth $h$ is for exponent $3 / 2$ always $20 \%$ with universal mathematical precision. This is totally independent of the material. For an assumed exponent 2 it would calculate to be 33.33\% [9] [10]. The non-consideration of such energy and thus also force losses is a violation of the first energy law in the ISO14577 standards and [3]! Our energy correction for directly depth related mechanical parameters is the factor 0.8 (4/5 ratio), for keeping with the first energy law. This is, of course, already implied in the indentation work $W_{\text {indent }}[10]$.

For example, motorized aviation with flying machines required new physical understanding of aerodynamics and also knowledge of materials' properties [11]. 
To make the former usable for airliners and airplanes they had to become faster, lighter, more efficient, and safer. The local analyses with (nano)indentation at the unphysical and iterating $\mathrm{H}_{\text {ISO }}$ and $\mathrm{E}_{\mathrm{r}-\mathrm{ISO}}$ level are unsuitable. They could therefore not prevent catastrophic failures (not only with airliners), which have been termed "failure by fatigue of materials". The liability clearly requires that local test procedures identify phase-transitions on the physical mathematic basis. We again urge ISO-ASTM to use the undeniable strict mathematical analyses, as presented here and in our cited publications since 2005. Addition of suitable ductilizers must optimize the super-alloys, so that the first phase transition onset force will be considerably above the permitted maximal force on them (also "pop-ins" must not occur upon load). Such analytical tests are required after the common long-term stretching, bending treatments, and after the repeated thermo-mechanical stress upon application with loading curves at the prescribed service intervals. The present technique is fast and easy for obtaining the onset information.

The penetration resistance $k\left[\mathrm{mN} / \mu \mathrm{m}^{3 / 2}\right]$ is literately the physical hardness with respect to the used indenter geometry. For the general applications the (effective) cone angle dependency of the self similar indenters is removed by the normalization as "penetration-resistance" hardness $H_{\text {phys }}=k / \pi \tan \alpha^{2}$, where $k$ of Equation (1) is energy corrected with factor 0.8 when the hardness shall be related to the indentation depth [9], and $\alpha$ is the effective cone angle [9].

Conversely, the still generally accepted definitions of indentation hardness as $H=F_{\mathrm{N}} / A_{\text {projected }}$ or $H_{\text {ISO }}=F_{\mathrm{N}} / A_{\text {contact }}$ use the entire maximal loading force for the depth. This seemed to verify the physically false exponent 2 . But the reasoning that the area of a cone "varies as the square of the depth of contact" [3] is misleading: such area $\left(\pi h^{2} \tan \alpha^{2}\right)$ variation is self evident, but the volume of the conical indenter varies with $\pi h^{3} \tan \alpha^{2} / 3$. Neither is the definition of indentation hardness according to ISO and [3] as "force (of a cone) over contact area" a "theoretical confirmation" of an "exponent 2" on $h$ as claimed in [12]. This definition severely violates the first energy law! $33.33 \%$ (for an exponent 2 ) of applied energy cannot be made out of nothing, and force is related to energy! Unfortunately, the energy violation remained apparently undiscovered for all of these authors since 1939 [4], and even so after our paper in 2013 [10] that quantified the violation with basic algebra. As above: the area of the $0-F_{\mathrm{N} \max }-h_{\max }$ triangle minus the area under the loading parabola with an exponent 2 would amount to one third of the total applied work. Nevertheless, this violation of the first energy law was not allowed to be literally expressed in publications before 1997 [9] [13]. ISO14577 and apparently most of the indentation world are urged to stop with tolerating the violations against basic physics.

A further advantage of the physical indentation resistance hardness $H_{\text {phys }}=$ $k / \pi \tan \alpha^{2}$ is its independence of the depth (self-similar indenter!). We can therefore for the first time choose from hardness with respect to the penetration act $(0.8 k)$ or with respect to the full indentation resistance (uncorrected $k$ ). An im- 
portant discussion on what should be used for what theoretical and practical use is now opened. The papers [9] [13], and [14] chose the first version for theoretical reasons. This can however easily be changed for more practical reasons. A similar choice between $F_{\mathrm{N} \max }$ or $0.8 F_{\mathrm{N} \max }$ applies for the definition of indentation elastic moduli $E_{\mathrm{r}}$ from the unloading curve. But none of such indentation moduli are the still claimed "Young's moduli". They resemble the bulk moduli [13]. Clearly, all hardness and moduli determinations must be at loads before the phase-transition onset and it can no longer be avoided to detect it with the Kaupp plot (1).

These important developments facilitated the easy detection of phase transitions by indentation. Previously such detection was restricted to a kink in the unloading curve. There is one in the unloading curve of silicon (though without onset information), which had been amply discussed as a particular exception [3]. On-site Raman spectroscopy, TEM, electrical resistance, electron diffraction, or micro-diffraction by synchrotron radiation revealed discontinuities and verified phase transition. We have a sharp kink upon Berkovich indentation onto silicon at the loading curve with onset information [1] [15]. Similar discontinuities in the Raman and current flow studies (see [15]) provided final support for our kink discontinuities as phase-transition onset. About 10 further examples using the more costly and highly specialized techniques are known. Correct indentation analysis obtains transitions right away with high frequency for all kinds of materials with transition onset and transition energy by simple indentation using Equation (1). Only the elucidation of the polymorph structures requires preferably onsite diffraction with highly focused synchrotron irradiation. Numerous confirmed phase transitions under hydrostatic pressure are long known, but these techniques are expensive and laborious without providing transition energies. Phase transition onset detections are indispensable for proper analyses of indentations.

A further application of Equation (1) with the penetration resistance $k$ is the reliability control of published measurements on the strict physical basis [8]. All partial deviations are either particular physical properties of the material (e.g. surface effects, phase changes, gradients, etc.) or experimental errors (e.g. poor calibration of force linearity, non-vertical indents, mix-up events, etc.). Importantly, the unphysical "exponent 2 on $h$ " claim for the loading parabola enforced several multi-parameter polynomial iterations that prevented data checks by wiping out all particular effects including the phase transitions. Only Equation (1) with its linear plots sorts out the special effects from published loading curves and detects deviations from unsuitable experimentation. These include too close indentations, false assignment of materials or polymorphs at large indents, integrations over phase transition onset discontinuities, false transition energy sign, and further flaws that are discussed below. These checks remove severe additional errors of published $H$ and $E_{\mathrm{r}}$ next to all other systematic errors, even with calibration standards. Some further types for disclosed errors can be 
found in the corresponding Section below.

Further applications use phase transition energies at different temperatures for the determination of phase transition activation energies [15]. Also multiple consecutive phase transitions have been published [14], and the transition energies of $\mathrm{SrTiO}_{3}$ on (011), $\alpha$-quartz on (010), InGaAs ${ }_{2}$ on (001) are already known [10]. However, the surface dependent anisotropy of phase transition energies awaited elucidation. Quartz is available in amorphous state and as hexagonal crystal it has different crystallographic faces. It is a particularly valuable material for that purpose. We describe the first anisotropies at four prominent crystal faces of $\alpha$-quartz.

\section{Materials and Methods}

A fully calibrated Hysitron Inc. Triboscope ${ }^{\circledR}$ Nanomechanical Test Instrument with $2 \mathrm{D}$ transducer and leveling device, connected to a Nanoscope AFM was used for the own indentations. The apex radii of the cube corner $(55 \mathrm{~nm})$ and Berkovich $(110 \mathrm{~nm})$ diamond indenter were directly measured by AFM in tapping mode. The leveling to $\pm 1^{\circ}$ was in $x$ and $y$ direction. Loading times were $30 \mathrm{~s}$ up to $5000 \mu \mathrm{N}$ final load. All our measurements were performed with the same cube corner with the effective cone angle of $\alpha=42.28^{\circ}$. The original data with about 1500 points each of our loading curves for $\alpha$-quartz (rock crystal) from [2] [6], and [7] were now used with more precise calculation (up to 10 significant figures) for the determination of the phase transition energies. Thus, rounding errors are minimized and Table 1 with many numbers characterize the specific data set as precisely as necessary. Loading data from the literature have been digitized with the Plot Digitizer 2.5.1 program (http://www.softpedia.com/). Electronic fittings or iterations whatsoever were never performed. The crystal structure data (P3(2)21, a 4.914, c 5.405) are from [16]. The crystal models were calculated using the Schakal 97 program [17].

A single well developed rock crystal with smooth surfaces and excellent colorless clarity was the $\alpha$-quartz sample without twins at the surface. Its indexed major faces were horizontally leveled to slopes of $\pm 1^{\circ}$ in $x$ and $y$ direction under AFM control at disabled plane-fit. All $F_{\mathrm{N}}$ and $h$ data pairs from the loading curves were loaded to Excel ${ }^{\circledR}$ (Microsoft; Redmond, USA, WA) for the calculation of the $h^{3 / 2}$ values and the linear branches of the regression lines provided the slopes (penetration resistances) $k_{1}$ and $k_{2}$, and the axis cuts $F_{1-\mathrm{a}}$ and $F_{2-\mathrm{a}}$. They were used with all of their figures for avoiding rounding errors. The linear regression coefficients $\mathrm{R}^{2}$ were in all cases $>0.999$ - 0.9999. The precise sharp intersection point (transition onset) was obtained by equalizing of the regression line equations and the so obtained $h_{\text {kink }}$ and $F_{\text {Nkink }}$ values were calculated by using Equation (2). All necessary terms are thus obtained, as $h_{\max }$ and $F_{\mathrm{N} m a x}$ are directly available.

$$
F_{\mathrm{N}}=k h^{3 / 2}+F_{1-\mathrm{a}} .
$$


Table1. Physical parameters from the cube corner indentations onto four different surfaces of $\alpha$-quartz (rock crystal) up to 5000 $\mu \mathrm{N}$ load.

\begin{tabular}{cccccccc}
\hline Entry & $\begin{array}{c}\text { Surface } \\
(\mathrm{hkl})\end{array}$ & $k_{1}\left(\mu \mathrm{N} / \mathrm{nm}^{3 / 2}\right)$ & $k_{2}\left(\mu \mathrm{N} / \mathrm{nm}^{3 / 2}\right)$ & $h_{\text {kink }}(\mathrm{nm})$ & $F_{\text {Nkink }}(\mu \mathrm{N} \mathrm{nm})$ & $\begin{array}{c}\Sigma \mathrm{W}_{\text {applied }} / 5000 \\
(\mu \mathrm{Nnm} / \mu \mathrm{N})\end{array}$ & $\begin{array}{c}W_{\text {trans }} / \mu \mathrm{N}^{(\mathrm{a})} \\
(\mu \mathrm{Nnm} / \mu \mathrm{N})\end{array}$ \\
\hline 1 & $(011)$ & 2.5443 & 1.8609 & 85.75601036 & 2097.594374 & 97.6134 & -15.744 \\
2 & $(010)$ & 2.1574 & 1.7169 & 105.8103095 & 2237.798772 & 101.3803 & -11.048 \\
3 & $(1-10)$ & 2.2037 & 1.6475 & 101.5669094 & 2264.183535 & 104.5936 & -14.663 \\
4 & $(101)$ & 2.2147 & 1.6773 & 100.3591866 & 2241.625060 & 103.4883 & -14.032 \\
\hline
\end{tabular}

${ }^{(a)}$ For practical reasons we do not use the factor 0.8 to the $\mu \mathrm{N}$ values for the normalizations from kink to the final force.

$$
\begin{gathered}
W_{1 \text {-applied }}=0.5 h_{\text {kink }}\left(F_{\text {N-kink }}+F_{1-\mathrm{a}} h_{\text {kink }}\right) . \\
W_{1-\text { indent }}=0.8 W_{1 \text {-applied }} \cdot \\
W_{2 \text {-indent }}=0.4 k\left(h^{5 / 2}-h_{\text {kink }}^{5 / 2}\right)+F_{2 \text {-a }}\left(h-h_{\text {kink }}\right) . \\
\text { full } W_{\text {applied }}=0.5 F_{\mathrm{N} \text {-max }} h_{\text {max }} . \\
W_{\text {transition }}=\text { full } W_{\text {applied }}-\Sigma\left(W_{\text {applied }}\right) .
\end{gathered}
$$

The used Equations (2)-(7) for the calculations contain all of the obvious corrections [14]. The meaning of the terms has already been explained. $F_{\text {a }}$ corrects for axis cuts of the regression lines that are due to surface effects. Only $F_{1-\mathrm{a}}$ is also influenced by the apical tip rounding radius $(R)$, giving larger penetration resistance up to $h_{\text {cone }}=R(1-\sin \alpha)$. It varies with the surface properties (including water layers). These depend on ambient conditions, which exclude their tabulation. The $W_{1 \text {-indent }}$ is calculated from $W_{1 \text {-applied }}$ according to Equation (4). $W_{2 \text {-indent }}$ must use integration and correction with $F_{2-\mathrm{a}}$ as in Equation (5), and $W_{2 \text {-applied }}$ is then obtained by multiplication with 1.25 in analogy to Equation (4). The balance of Equation (6) and Equation (7) gives the transition energy $W_{\text {trans }}$ that was reasonable rounded in Table 1 .

\section{Results and Discussion}

\subsection{Quartz, Aluminium, Tungsten, and Sapphire as Calibration Standards of ISO}

Unfortunately, all indentations of the most cited publication of Oliver-Pharr in 1992 [3] with their iterated values of hardness $H$ and reduced elastic modulus $E_{\mathrm{r}}$ (from there with Poisson's ratio elastic modulus $E$, which has been unduly called "Young's modulus" [13]). They became standards for instrument calibration and numerous further quantities of materials for iterations and finite element calculations. It appears therefore of primary importance to check the validity of these old though still used measurements that lacked the 1992 not available physical insights. The authors of [3] did not have universal Equation (1) and thus missed that their force linearity and so their instrument compliance were not well adjusted above $90 \mathrm{mN}$ to $120 \mathrm{mN}$ load for their published curves as designated with 
aluminium (their Figure 4), "quarts" on (001) (their Figure 5), sapphire (their Figure 7), and tungsten (their (Figure 9). That is consistently revealed by strong positive deviations from linearity (without kink appearance) above about $90 \mathrm{mN}$ in our Kaupp plots (1). Defective tip surface cannot be the reason, as these deviations occur at different depths $h$. There is only one exception in [3]: the Kaupp plot of their Figure 6 for soda lime glass has the last branch staying linear up to $120 \mathrm{mN}$ load. Nevertheless, the iterated standard values of hardness $H$ and modulus $E_{\mathrm{r}}$ that use the maximal force are in error for all 5 examples: the faulty calibration adds to the unphysical $h^{2}$, the energy law violation, and the non-consideration of the phase transition onsets that occur before that load. Not the pristine material is tried to be characterized! These errors perpetuate in all further iterations that are made to converge to these values and the numerous qualities that are deduced from all of these values. They have at least influenced various reference table entries, not to speak of finite element calculations. Particularly troublesome are the errors in [3] for aluminium, which is often used as a standard for microindentation. Its phase change onset under Berkovich is known to be at $30-40$ $\mathrm{mN}$, which became first known in 2013 [2]. And there are further errors in [3]. The designation of their Figure 8 in [3] with "fused quartz" is in error. It reveals three linear branches $\left(k_{1}=76.044, k_{2}=109.12\right.$, and $k_{3}=123.3 \mathrm{mN} / \mu \mathrm{m}^{3 / 2}$, all with $\left.R^{2}>0.999\right)$ when plotted with Equation (1). This corresponds so perfectly with the soda lime glass values from their Figure 6 of [3] $\left(k_{1}=77.909, k_{2}=105.28\right.$, and $k_{3}=122.0 \mathrm{mN} / \mu \mathrm{m}^{3 / 2}$ ) that the claimed "fused quartz" curve must in fact belong to the result from another soda lime glass indentation, despite the about $250 \mathrm{~nm}$ different maximal depths. Furthermore, their Figure 5 in [3] of crystalline quartz on (001) is totally misinterpreted. The authors do not realize the situation from 0 to $5 \mathrm{mN}$ loads that is not really discernible in the Kaupp plot (1) up to $120 \mathrm{mN}$. As expected for crystalline quartz it rightfully starts steeply and soon thereafter continues less steeply, apparently by producing fused quartz around the Berkovich with its then endothermic phase transition. The initial exothermic $\alpha$-quartz transition [6] [7] can however not be analyzed in more detail at that loading range without a large number of original data points. But it is extremely troublesome to the reader of their Figure 5 in [3], who became misguided about the mechanical properties of crystalline quartz until [6] and [7] had appeared. Clearly, the indentation of fused quartz up to $120 \mathrm{mN}$ deserves new investigation. The exothermic $\alpha$-quartz indentations ending at $5 \mathrm{mN}$ are discussed in Table 1. Importantly, the properties of tungsten $\left(k_{1}=95.57, k_{2}=\right.$ $114.50 \mathrm{mN} / \mu \mathrm{m}^{3 / 2}$; kink $\left.\approx 35.6 \mathrm{mN}\right)$ and sapphire on (0001) $\left(k_{1}=236.58\right.$ and $k_{2}=$ $264.68 \mathrm{mN} / \mathrm{mm}^{3 / 2}$, kink $\approx 31.5 \mathrm{mN}$ ) (the better older curve up to $90 \mathrm{mN}$ load of [18] was used) are poor calibration standards for the harder materials without consideration of their phase transitions. Further phase transitions are detected in macro-indentations for example sapphire transforms also at about $12 \mathrm{~N}$ load and $5.9 \mu \mathrm{m}$ depth [2], and so does soda-lime glass at about $14 \mathrm{~N}$ at $11.7 \mu \mathrm{m}$ when the loading curves with a Vickers indenter of [19] are analyzed with the Kaupp plot 
(1), and we mention here to the four consecutive phase transition onsets of $\mathrm{NaCl}$ at $0.003397,2.487,9.1186$, and $24.4284 \mathrm{~N}$ loads [14]. Any non-consideration of phase transitions generates errors at hardness and modulus iterations, including other materials and finite element calculations as these values concern not detected polymorphs, but not the pristine material. We complain that apparently nobody else did check all these grave inconsistencies since 1992 and hope that the new physical insights and possibilities will be used, instead of violating basic physics.

\subsection{Fused Quarts, Transition Energy}

Fused quartz is the most used calibration standard for nanoindentations. We analyze therefore the corresponding Berkovich (with half angle $\theta$ of $65.3^{\circ}$ ) loading curves of prominent instrument provider Handbooks with respect to the Equations (1)-(7) and use of the new applications without any iteration. The first point is the detection of the long known amorphous to amorphous phase transition [20] under indentation [1], because hardness and modulus of pristine materials must be determined at loads before the first phase transition onset. The early onset of this endothermic transition $\left(k_{2}>k_{1}\right)$ has been denied on the basis of poor, or fitted, or too extended curves with low precision (e.g. [12], and many others), but a more detailed analysis with the Kaupp plot (1) reveals the transition as long as these are experimental [14] and so do the excellent loading curves from instrument builders as analyzed in [1]. However, the energetics of such transition is still unknown. The calculation is therefore performed by using the loading curves as published in the TriboScope manuals of Hysitron and of CISCO for UMIS. Both linearized loading curves give two linear branches that correlate with $\mathrm{R}^{2}=0.9999$, each upon regression with the Kaupp plot (1). Their penetration resistance values are for $k_{1} 1.9654$ and 1.9672 and those for $k_{2}$ are 2.4392 and $2.3936 \mu \mathrm{N} / \mathrm{nm}^{3 / 2}$ for Hysitron and CISCO, respectively. This leads after surface-cut corrections to transition onset depths of 109.6476605 and 100.682078 $\mathrm{nm}$ at 2.348 and $2.117 \mathrm{mN}$. These differences reflect different measurement conditions. Most likely are different force calibrations or horizontal sample leveling devices that are not specified. It is therefore not surprising that the calculated normalized per $\mu \mathrm{N}$ transition energy values also differ: we calculate 6.206 and $3.563 \mu \mathrm{Nnm} / \mu \mathrm{N}$, respectively, for the endothermic transitions. These values are remarkably large when compared with the exothermic transitions that give the negative values of crystalline quartz (Table 1).

\section{3. $\alpha$-Quartz, Transition Energies at Different Faces}

Unlike fused quartz, crystalline $\alpha$-quartz in the form of rock crystal undergoes exothermic phase transition upon sufficient indentation stress. The projected images of the studied surfaces are shown in Figure 1. Anisotropic behavior for the transition energies upon cube-corner indentation is to be expected, as the crystal packing is different. In particular the penetration resistances $k_{1}$ and $k_{2}$ had already been shown to be anisotropic with different indentation works $W_{\text {indent }}$ 
[2]. Table 1 with Miller indices (hkl) for simplicity (as the "i" in (hkil) is redundant) repeats less rounded $k$ and kink values. The structure of the indented surface is important for the understanding of the varying $W_{\text {trans }}$ values of Table 1. Channels will facilitate penetration the better these are vertically aligned. That is roughly reflected by the $k_{1}, h_{\text {kink }}$, and $W_{\text {trans }} / \mu \mathrm{N}$ values (entries 1 and 2 are most different, 3 and 4 are in between), but not by $k_{2}, F_{\text {Nkink}}$, and $\Sigma \mathrm{W}_{\text {applied }} / \mu \mathrm{N}$. Clearly, there is also the force that acts normal to the surface of the cube corner with the opposite of its half angle $\theta=35.26^{\circ}$. It is therefore important to also consider the location of channels exiting from the side faces at the indenter surface for the transition energies. We therefore construct such surfaces at $35^{\circ}$ and assume that not all of their so seen shapes will be destructed while the cube corner penetrates. They are obtained by rotation of the crystal structure around the $\mathrm{X}$ axis by + and $-35^{\circ}\left(\mathrm{rX} \pm 35^{\circ} \equiv 180^{\circ} \pm 35^{\circ}\right)$ and the same around an $\mathrm{Y}$ axis (as $\mathrm{rY} \pm 35^{\circ} \equiv$ $180^{\circ} \pm 35^{\circ}$ ). The resulting images are projected (Figure 2 and Figure 3 ) and visually analyzed. The differences on the determined circumventing skew faces prove large enough to be helpful for the understanding of the different results. It turns out that the highest amount of normalized exothermic energy $W_{\text {trans }}=$ $-15.744 \mu \mathrm{Nnm} / \mu \mathrm{N}$ is produced by indentation upon the (011) surface (entry 1 ) of $\alpha$-quartz with a cube corner indenter. The (011) packing exhibits not very favorable skew channels, as can be best seen in the center of the image where the

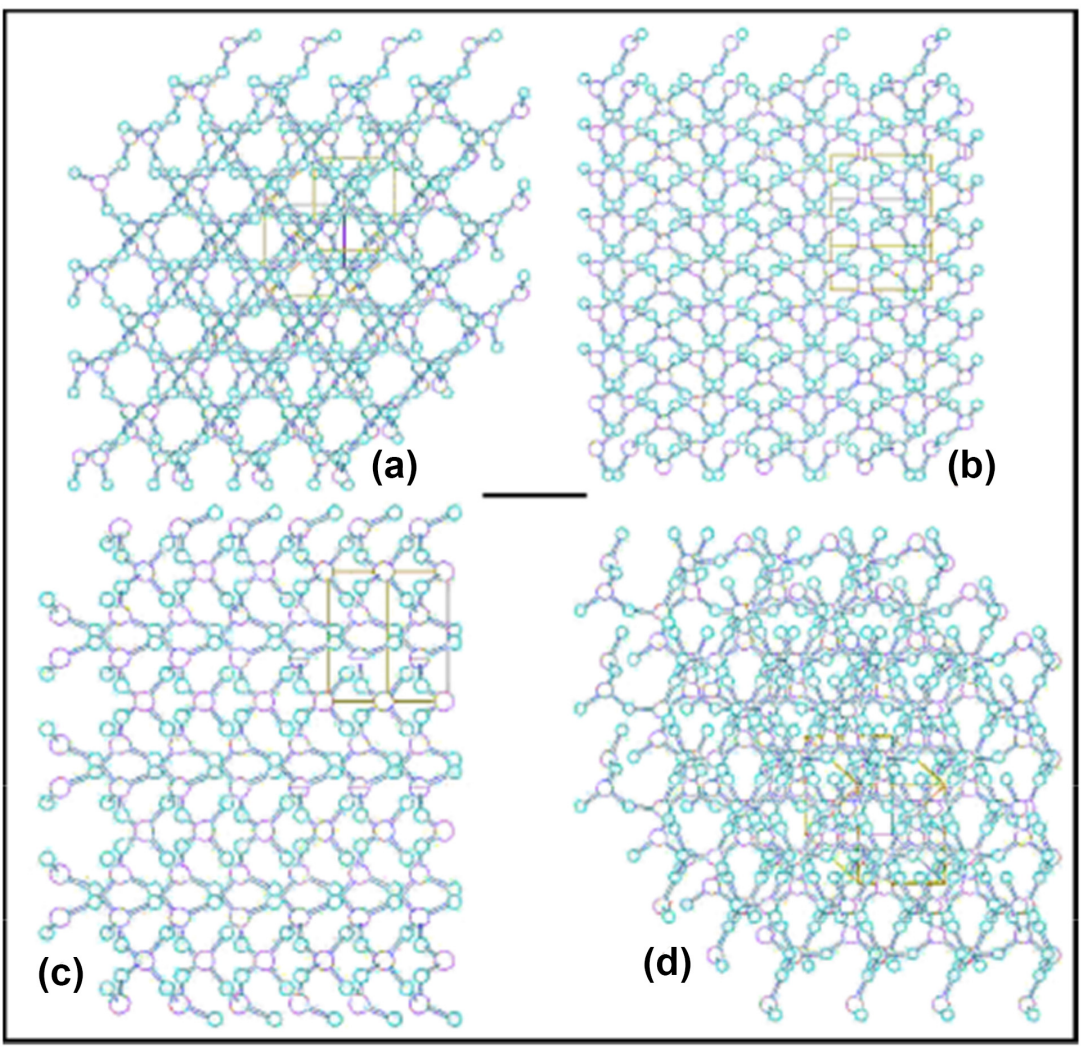

Figure 1. Surface projections on $\alpha$-quartz with four different tetrahedrons upon each other in the center areas; (a) (011); (b) 010); (c) (1-10); (d) (101); the bar corresponds to 5 Å length. 


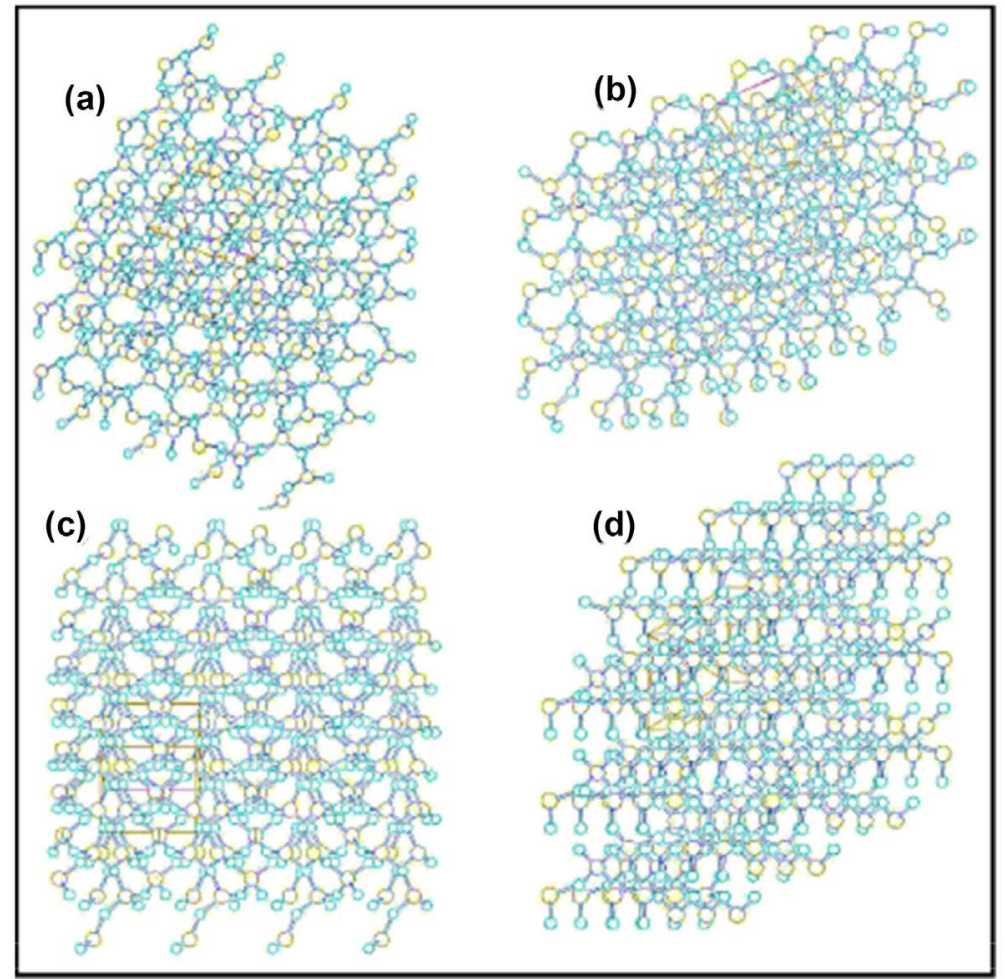

Figure 2. $\alpha$-Quartz $35^{\circ}$ skew side faces under the (011) surface ; (a) $\mathrm{rX} 35^{\circ}$; (b) $\mathrm{rX}-35^{\circ}$; (c) $\mathrm{rY} 35^{\circ}$; (d) $\mathrm{rY}-35^{\circ}$.

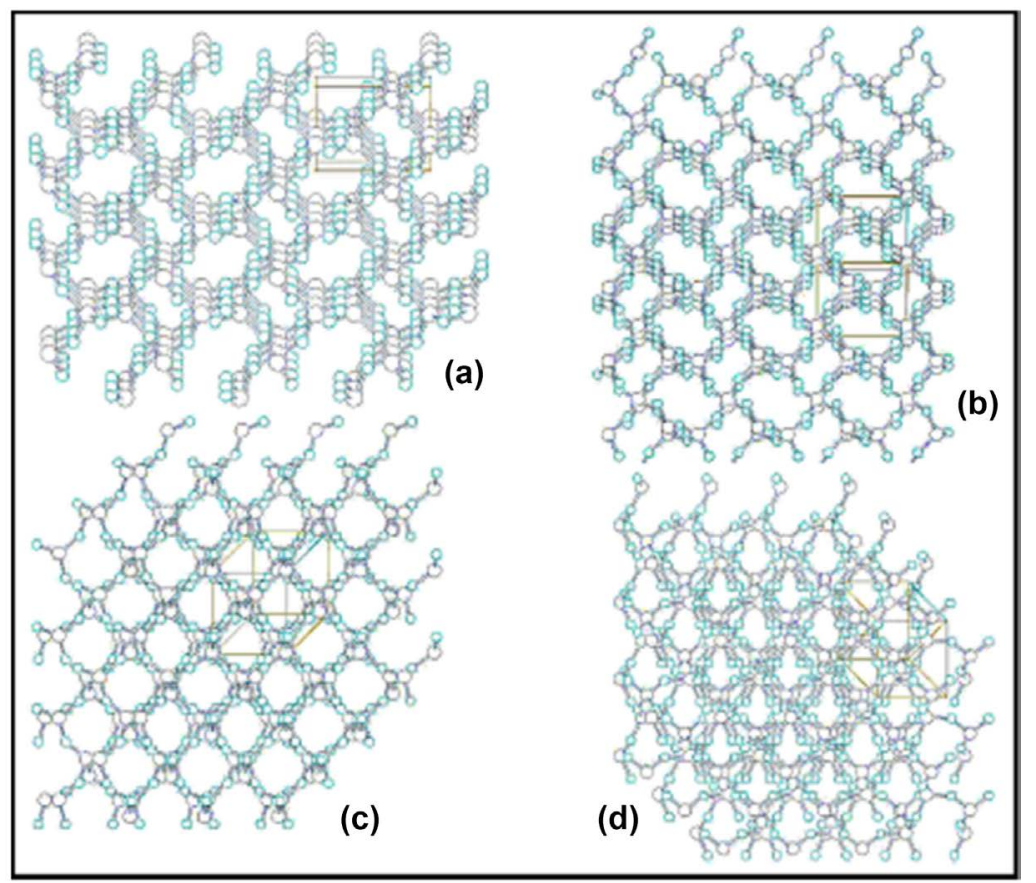

Figure 3. $\alpha$-Quartz $35^{\circ}$ skew side faces under the (010) surface; (a) $\mathrm{rX} 35^{\circ}$; (b) $\mathrm{rX}-35^{\circ}$; (c) $\mathrm{rY} 35^{\circ}$; (d) rY $-35^{\circ}$.

view goes through 5 of the interlocked pyramidal layers (Figure 1(a)). The normal indentation depth for the phase transition onset $h_{\text {kink }}$ and $F_{\text {Nkink }}$ are the least 
of all studied cases and the required work down to the transition onset force is lower than with the other surfaces. Furthermore, Figure 2 shows four of the eight $35^{\circ}$ skew side structures around the cube corner under (011). In that case only comparatively small channels are available all around the cube corner. Therefore, the exothermic phase-transition is produced in a highly concentrated manner. This does not cost much displacement energy. It just detracts least from the transition energy, leaving more for it.

Consistently, the least exothermic transition energy among the tested surfaces is produced under the (010) surface (entry 2) with $-11.0485 \mu \mathrm{Nnm} / \mu \mathrm{N}$. It exhibits straight channels (Figure 1(b)). The penetration down to the phase transition onset is deep and the required onset energy from $F_{\text {Nkink }}$ is high (Table 1). The cube corner is surrounded by large $35^{\circ}$ skew faces with almost orthogonal channels that are well shaped for the transport of materials (Figure 3). Such materials transports cost energy, which detracts from the exothermic transition energy. This certainly helps to understand the reasons for the extremes under the (011) and (010) surfaces. Consistently, the normalized transition energies (under (1-10) and (101) indentations (entries 3 and 4) are between these extremes. They penetrate almost with the similar depth of entry 2 and their $35^{\circ}$ skew side walls (not shown here) are less favorable under (011) and (010). Thus, their normalized $W_{\text {trans }}$ values are almost equal and between the extremes (Table 1). Clearly, the complicated variations of the normalized anisotropic transition energies require the whole anisotropic 3D packing of the crystal. All qualities of Table 1 interact. This has to be taken into account for all crystals and other non-isotropic materials.

\section{Conclusion}

The universal Equations (1)-(7) are physically and mathematically deduced beyond any doubt for vertical indentations with self-similar indenters [8] [10]. All depend on the physical exponent $3 / 2$ on $h$ rather than on the assumed exponent 2 that requires iterations with violation of the first energy law. The now possible detections of phase transition onsets and phase transition energies are indispensable for the characterization of materials and proper analyses of indentations. They are of theoretical and practical importance. Their unprecedented anisotropy deserves consideration with further crystals, not uniform materials, and composites for a better understanding of their failures. Numerous further studies on those lines are therefore essential. We used the iteration-less physical analysis of common normal force-depth parabolas not only for data checks, but also for the detection of phase transitions under load. These include the most cited force-depth curves in [3]. Unfortunately these data of Oliver Pharr were taken as the basis for ISO14577, without knowing of their force calibration errors and the further errors in the absence of the physical data check possibilities from Equation (1). Their definition of $H_{\text {ISO }}$ and $E_{\text {r-ISO }}$ does not consider phase transitions under load and they violate the first energy law. For example [21] fit- 
ted the incorrect data for aluminium and tungsten, and others continued with fitting of loading curves without considering initial effects, phase transitions, and other particularities. All of these errors with iterations since 1992 must be corrected with physically sound analyses. We tell here how the various and obvious errors of the iterative indentation treatments are avoided: the closed formulas (1)-(7) for the calculations are presented and used at characteristic examples. They rely on excellent linear correlations, contain the corrections for initial effects, and do not violate the first energy law. Unfortunately, there was no other protest against the first energy law violation for 70 years, even though the remaining pressure for elastic moduli and long-range plasticization were always known and discussed. Surprisingly enough, it was not asked from where the necessary force and energy might come from. We continue to urge ISO for changing its 14,577 standard, so that the very common phase transitions upon indentations do not longer stay undetected, etc. It is certainly good scientific practice to consider the physically enforced formulas (1)-(7). Their perhaps most important advances are the unprecedented applications of nano-, micro- and macro-indentations without violating the first energy law. We cannot live with [3] and ISO followers who still want to "produce" the pressure plus plasticization work from nothing. Only the iteration-free physical formulas are able to obtain reliable materials' properties, including the phase transitions and their energies under load. That applies to crystalline, amorphous, and plastic materials under mechanical stress that must always be smaller than the phase transition stress for avoiding failure of materials in daily life, not to speak of liability problems. Clearly, first order phase transformations produce polymorph's interfaces that increase the probability for cracking [14]. Reversibility of phase transitions upon pressure release is more likely for the endothermic transitions than for exothermic ones. It has already been shown that the activation energies of phase transitions can be obtained by temperature dependent indentations with their phase transitions [15]. So this appears particularly important for the choice of proper materials that are stressed by both load and temperature. That will be particularly important for the field of super alloys [10]. Such measurements are easily and cheaply available with presently existing instrumentation.

\section{Conflicts of Interest}

The author declares no conflicts of interest regarding the publication of this paper.

\section{References}

[1] Kaupp, G. and Naimi-Jamal, M.R. (2010) The Exponent 3/2 at Pyramidal Nanoindentations. Scanning, 32, 265-281. https://doi.org/10.1002/sca.20206

[2] Kaupp, G. and Naimi-Jamal, M.R. (2013) Penetration Resistance and Penetrability in Pyramidal (Nano)Indentations. Scanning, 35, 88-111.

https://doi.org/10.1002/sca.21038 
[3] Oliver, W.C. and Pharr, G.M. (1992) An Improved Technique for Determining Hardness and Elastic Modulus Using Load and Displacement Sensing Indentation Experiments. Journal of Materials Research, 7, 1564-1583. https://doi.org/10.1557/JMR.1992.1564

[4] Love, A.E.H. (1939) Boussinesq's Problem for a Rigid Cone. The Quarterly Journal of Mathematics (Oxford), 10, 161-175. https://doi.org/10.1093/qmath/os-10.1.161

[5] Sneddon, I.N. (1965) The Relation between Load and Penetration in the Axisymmetric Boussinesq Problem for a Punch of Arbitrary Profile. International Journal of Engineering Science, 3, 47-57. https://doi.org/10.1016/0020-7225(65)90019-4

[6] Naimi-Jamal, M.R. and Kaupp, G. (2005) Quantitative Evaluation of Nanoindents: Do We Need More Reliable Mechanical Parameters for the Characterization of Materials? International Journal of Materials Research, 11, 1226-1236.

[7] Kaupp, G. (2006) Atomic Force Microscopy, Scanning Nearfield Optical Microscopy and Nanoscratching-Application to Rough and Natural Surfaces. Springer, Berlin-Heidelberg, New York.

[8] Kaupp, G. (2016) The Physical Foundation of $F_{\mathrm{N}}=k h^{3 / 2}$ for Conical/Pyramidal Indentation Loading Curves. Scanning, 38, 177-179. https://doi.org/10.1002/sca.21223

[9] Kaupp, G. (2017) The ISO Standard 14577 for Mechanics Violates the First Energy Law and Denies Physical Dimensions. Journal of Materials Science and Engineering, 6, 321-328. https://doi.org/10.4172/2169-0022.1000321

[10] Kaupp, G. (2013) Penetration Resistance: A New Approach to the Energetics of Indentations. Scanning, 35, 392-401.

[11] Wright, W. (1906) Flying-Machine. US Patent No. 821393.

[12] Merle, B., Maier, V. and Durst, K. (2014) Experimental and Theoretical Confirmation of the Scaling Exponent 2 in Pyramidal Load Displacement Data for Depth Sensing Indentation. Scanning, 36, 526-529. https://doi.org/10.1002/sca.21151

[13] Kaupp, G. (2017) Challenge of Industrial High-Load One-Point Hardness and of Depth Sensing Modulus. Journal of Materials Science and Engineering, 6, 348-355.

[14] Kaupp, G. (2018) Six Polymorphs of Sodium Chloride upon Depth-Sensing Scanning Macroindentatiom with Unusual Long-Range Cracks Requiring 30 N Load. Journal of Materials Science and Engineering, 7, 473-483. https://doi.org/10.4172/2169-0022.1000473

[15] Kaupp, G. (2014) Activation Energy of the Low-Load NaCl Transition from Nanoindentation Loading Curves. Scanning, 36, 582-589. https://doi.org/10.1002/sca.21158

[16] Smith, G.S. and Alexander, L.E. (1963) Refinement of the Atomic Parameters of a-Quartz. Acta Crystallographica, 16, 462-471. https://doi.org/10.1107/S0365110X63001298

[17] Keller, E. (1997) Schakal 97. Kristallographisches Institut der Universität Freiburg i. $\mathrm{Br}$.

[18] Page, T.F., Oliver, W.C. and McHargue, C.J. (1992) The Deformation Behavior of Ceramic Crystals Subjected to Very Low Load (NanoIndentations). Journal of Materials Research, 7, 450-473. https://doi.org/10.1557/JMR.1992.0450

[19] Thurn, J., Morris, D.J. and Cook, R.F. (2002) Depth-Sensing Indentation at Macroscopic Dimensions. Journal of Materials Research, 17, 2679-2690. https://doi.org/10.1557/JMR.2002.0388 
[20] Trachenko, K. and Dove, M. (2003) Intermediate State in Pressurized Silica Glass: Reversibility Window Analogue. Physical Review B, 67, 212203/1-212203/3. https://doi.org/10.1103/PhysRevB.67.212203

[21] Cheng, Y.T. and Cheng, C.M. (1998) Further Analysis of Indentation Loading Curves: Effects of Tip rounding on Mechanical Property Measurements. Journal of Materials Research, 13, 1059-1064. https://doi.org/10.1557/JMR.1998.0147 